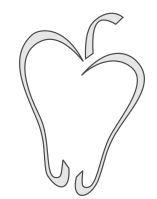

ISSN $1852-4249$

\title{
A note on the consensus time of mean-field majority-rule dynamics
}

\author{
Damián H. Zanette ${ }^{1 *}$
}

\begin{abstract}
In this work, it is pointed out that in the mean-field version of majority-rule opinion dynamics, the dependence of the consensus time on the population size exhibits two regimes. This is determined by the size distribution of the groups that, at each evolution step, gather to reach agreement. When the group size distribution has a finite mean value, the previously known logarithmic dependence on the population size holds. On the other hand, when the mean group size diverges, the consensus time and the population size are related through a power law. Numerical simulations validate this semi-quantitative analytical prediction.
\end{abstract}

Much attention has been recently paid, in the context of statistical physics, to models of social processes where ordered states emerge spontaneously out of disordered initial conditions (homogeneity from heterogeneity, dominance from diversity, consensus from disagreement, etc.) [1]. Not unexpectedly, many of them are adaptations of well-known models for coarsening in interacting spin systems, whose dynamical rules are reinterpreted in the framework of social-like phenomena. The voter model $[2,3]$ and the majority rule model $[4,5]$ are paradigmatic examples. In the latter, consensus in a large population is reached by accumulative agreement events, each of them involving just a group of agents. The present note is aimed at briefly revisiting previous results on the time needed to reach consensus in majority-rule dynamics, stressing the role of the size distribution of the involved groups. It is found that the growth of the consensus time with the population size shows

*E-mail: zanette@cab.cnea.gov.ar

1 Consejo Nacional de Investigaciones Científicas y Técnicas, Centro Atómico Bariloche and Instituto Balseiro, 8400 San Carlos de Bariloche, Río Negro, Argentina. distinct behaviors depending on whether the mean value of the group size distribution is finite or not.

Consider a population of $N$ agents where, at any given time, each agent has one of two possible opinions, labeled +1 and -1 . At each evolution step, a group of $G$ agents ( $G$ odd) is selected from the population, and all of them adopt the opinion of the majority. Namely, if $i$ is one of the agents in the selected group, its opinion $s_{i}$ changes as

$$
s_{i} \rightarrow \operatorname{sign} \sum_{j} s_{j},
$$

where the sum runs over the agents in the group. Of course, only the agents, not the majority, effectively change their opinion. In the mean-field version of this model, the $G$ agents selected at each step are drawn at random from the entire population.

It is not difficult to realize that the mean-field majority-rule (MFMR) dynamics is equivalent to a random walk under the action of a force field. For a finite-size population, this random walk is moreover subject to absorbing boundary conditions. Think, for instance, of the number $N_{+}$of agents with opinion +1 . As time elapses, $N_{+}$changes randomly, with transition probabilities that depend on $N_{+}$ itself, until it reaches one of the extreme values, 
$N_{+}=0$ or $N$. At this point, all the agents have the same opinion, the population has reached full consensus, and the dynamics freezes.

In view of this overall behavior, a relevant quantity to characterize MFMR dynamics in finite populations is the consensus time, i.e. the time needed to reach full consensus from a given initial condition. In particular, one is interested in determining how the consensus time depends on the population size $N$. The exact solution for three-agent groups $(G=3)[5]$ shows that the average number of steps needed to reach consensus, $S_{c}$, depends on $N$ as

$$
S_{c} \propto N \log N,
$$

for large $N$. The proportionality factor depends in turn on the initial unbalance between the two opinions all over the population. The analogy of MFMR dynamics with random walks suggests that this result should also hold for other values of the group size $G$, as long as $G$ is smaller than $N$. This can be easily verified by solving a rate equation for the evolution of $N_{+}$[1]. Numerical results and semi-quantitative arguments [6] show that Eq. (2) is still valid if, instead of being constant, the value of $G$ is uniformly distributed over a finite interval.

What would happen, however, if, at each step, $G$ is drawn from a probability distribution $p_{G}$ that allows for values larger than the population size? If, at a given step, the chosen group size $G$ is equal to or largen than $N$, full consensus will be instantly attained and the evolution will cease. In the randomwalk analogy, this step would correspond to a single long jump taking the walker to one of the boundaries. Is it possible that, for certain forms of the distribution $p_{G}$, these single large- $G$ events could dominate the attainment of consensus? If it is so, how is the $N$-dependence of the consensus time modified?

To give an answer to these questions, assume that $G$ is drawn from a distribution which, for large $G$, decays as

$$
p_{G} \sim G^{-\gamma},
$$

with $\gamma>1$. Tuning the exponent $\gamma$ of this powerlaw distribution, large values of $G$ may become sufficiently frequent as to control consensus dynamics.
The probability that at the $S$-th step the selected group size is $G \geq N$, while in all preceding steps $G<N$, reads

$$
P_{S}=\left(\sum_{G=G_{\min }}^{N-1} p_{G}\right)^{S-1} \sum_{G=N}^{\infty} p_{G},
$$

where $G_{\min }$ is the minimal value of $G$ allowed for by the distribution $p_{G}$. The average waiting time (in evolution steps) for an event with $G \geq N$ is thus

$$
S_{w}=\sum_{S=1}^{\infty} S P_{S}=\left(\sum_{G=N}^{\infty} p_{G}\right)^{-1} \propto N^{\gamma-1},
$$

where the last relation holds for large $N$ when $p_{G}$ verifies Eq. (3).

Compare now Eqs. (2) and (5). For $\gamma>2$ (respectively, $\gamma \leq 2)$ and asymptotically large population sizes, one has $S_{w} \gg S_{c}$ (respectively, $S_{w} \ll$ $S_{c}$ ). This suggests that above the critical exponent $\gamma_{\text {crit }}=2$, the attainment of consensus will be driven by the asymptotic random-walk features that lead to Eq. (2). For smaller exponents, on the other hand, consensus will be reached by the occurrence of a large- $G$ event, in which all the population is entrained at a single evolution step. Note that $\gamma_{\text {crit }}$ stands at the boundary between the domain for which the mean group size is finite $\left(\gamma>\gamma_{\text {crit }}\right)$ and the domain where it diverges $\left(\gamma<\gamma_{\text {crit }}\right)$.

In order to validate this analysis, numerical simulations of MFMR dynamics have been performed for population sizes ranging from $10^{2}$ to $10^{5}$. The probability distribution for the group size $G$ has been introduced as follows. First, define $G=2 g+1$. Choosing $g=1,2,3, \ldots$ ensures that the group size is odd and $G \geq 3$. Then, take for $g$ the probability distribution

$$
p_{g}=\frac{1}{\zeta(\gamma)} g^{-\gamma},
$$

where $\zeta(z)$ is the Riemann zeta function. With this choice, $p_{G}$ satisfies Eq. (3). The average waiting time for a large- $G$ event, given by Eq. (5), can be exactly given as

$$
S_{w}=\frac{\zeta(\gamma)}{\zeta(\gamma, 1+N / 2)},
$$


where $\zeta(z, a)$ is the generalized Riemann (or Hurwitz [7]) zeta function. In the numerical simulations, both opinions were equally represented in the initial condition. The total number of steps needed to reach full consensus, $S$, was recorded and averaged over series of $10^{2}$ to $10^{6}$ realizations (depending on the population size $N$ ).

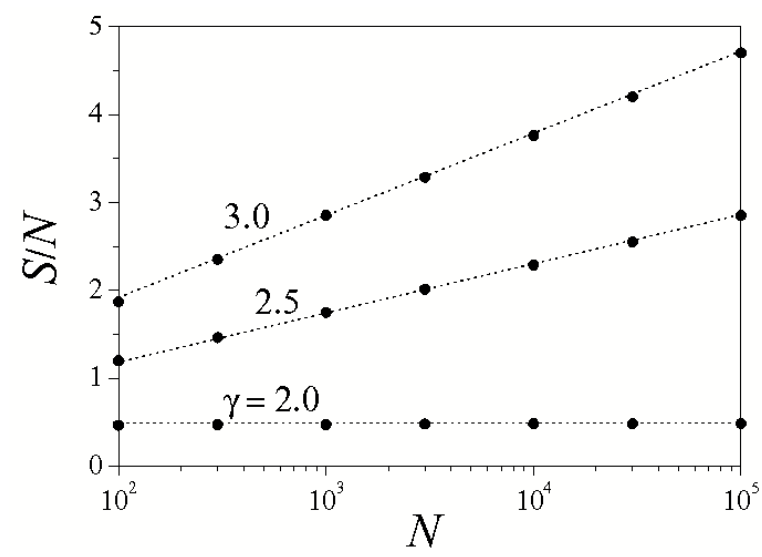

Figure 1: Numerical results for the number of steps needed to reach consensus, $S$, normalized by the population size $N$, as a function of $N$, for three values of the exponent $\gamma$. The straight dotted lines emphasize the validity of Eq. (2) for $\gamma=2.5$ and 3 . For $\gamma=2$ the line is horizontal, suggesting $S \propto N$.

The two upper data sets in Fig. 1 show the ratio $S / N$ for two values of the exponent $\gamma>\gamma_{\text {crit }}$. Since the horizontal scale is logarithmic, a linear dependence in this graph corresponds to the proportionality given by Eq. (2). Dotted straight lines illustrate this dependence. For these values of $\gamma$, therefore, the relation between the consensus time and the population size coincides with that of the case of constant $G$. For the lowest data set, which corresponds to $\gamma=\gamma_{\text {crit }}$, the relation ceases to hold. The horizontal dotted line suggests that now $S \propto N$, as predicted for $\gamma=2$ by Eq. (5).

The log-log plot of Fig. 2 shows the number of steps to full consensus as a function of the population size for three exponents $\gamma \leq \gamma_{\text {crit }}$. The dotted straight line has unitary slope, representing the proportionality between $S$ and $N$ for $\gamma=2$. For lower exponents, the full curves are the graphic representation of $S_{w}$ as given by Eq. (5). The excellent

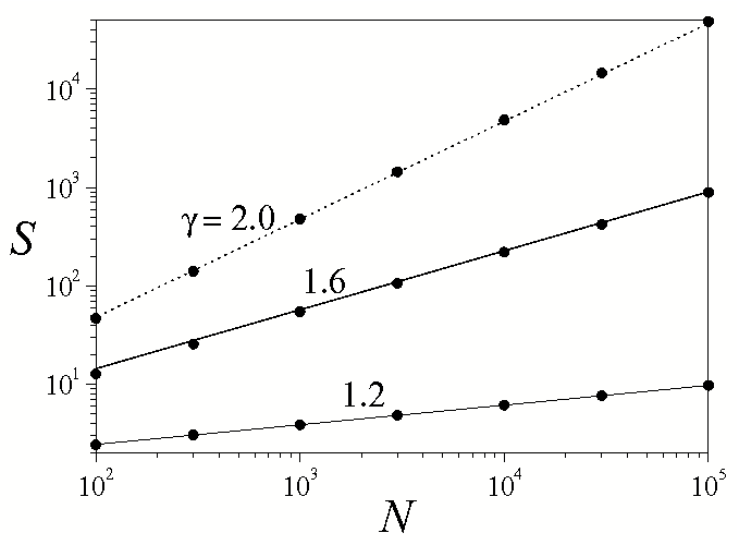

Figure 2: Number of steps needed to reach consensus as a function of the population size, for three values of the exponent $\gamma$. The slope of the straight dotted line equals one. Full curves correspond to the function $S_{w}$ given in Eq. (7).

agreement between $S_{w}$ and the numerical results for $S$ demonstrates that, for these values of $\gamma$, the consensus time in actual realizations of the MFMR process is in fact dominated by large- $G$ events.

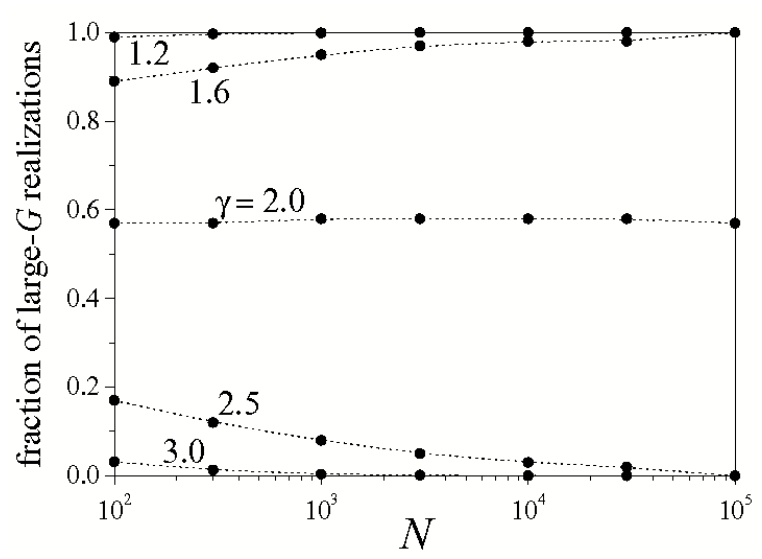

Figure 3: Fraction of realizations where consensus is attained through a large- $G$ event as a function of the population size, for several values of the exponent $\gamma$.

A further characterization of the two regimes of consensus attainment is given by the fraction of realizations where consensus is reached through a 
Papers in Physics, vol. 1, ART. 010002 (2009) / D. H. Zanette

large- $G$ event. This is shown in Fig. 3 as a function of the population size. For $\gamma<\gamma_{\text {crit }}$, consensus is the result of a step involving the whole population in practically all realizations. As $N$ grows, the frequency of such realizations increases as well. The opposite behavior is observed for $\gamma>\gamma_{\text {crit }}$. For the critical exponent, meanwhile, the fraction of large$G$ realizations is practically independent of $N$, and fluctuates slightly around 0.57 .

In summary, it has been shown here that in majority-rule opinion dynamics, the dependence of the consensus time on the population size exhibits two distinct regimes. If the size distribution of the groups of agents selected at each evolution step decays fast enough, one reobtains the logarithmic analytical result for constant group sizes. If, on the other hand, the distribution of group sizes decays slowly, as a power law with a sufficiently small exponent, the dependence of the consensus time on the population size is also given by a power law. The two regimes are related to two different mechanisms of consensus attainment: in the second case, in particular, consensus is reached during events which involve the whole population at a single evolution step. The logarithmic regime occurs when the mean group size is finite, while in the power-law regime the mean value of the distribution of group sizes diverges. In connection with the random-walk analogy of majority-rule dynamics, this is reminiscent of the contrasting features of standard and anomalous diffusion [8].
[1] C Castellano, S Fortunato, V Loreto, Statistical physics of social dynamics, Rev. Mod. Phys. 81, 591 (2009).

[2] M Scheucher, H Spohn, A soluble kinetic model for spinodal decomposition, J. Stat. Phys. 53, 279 (1988).

[3] P L Krapivsky, Kinetics of a monomermonomer model of heterogeneous catalysis, Phys. Rev. A 45, 1067 (1992).

[4] S Galam, Minority opinion spreading in random geometry, Eur. Phys. J. B 25, 403 (2002).

[5] P L Krapivsky, S Redner, Dynamics of majority rule in two-state interacting spin systems, Phys. Rev. Lett. 90, 238701 (2003).

[6] C J Tessone, R Toral, P Amengual, H S Wio, M San Miguel, Neighborhood models of minority opinion spreading, Eur. Phys. J. B 39, 535 (2004).

[7] J Spanier, K B Oldham, The Hurwitz Function $\zeta(\nu ; u)$, In: An Atlas of Functions, pag. 653 Hemisphere, Washington, DC (1987).

[8] U Frisch, M F Shlesinger, G Zaslavsky, Eds. Lévy Flights and Related Phenomena in Physics, Springer, Berlin (1995). 